\title{
Some Recent Results with CLAS
}

\author{
M. Holtrop* and for the CLAS Collaboration ${ }^{\dagger}$ \\ ${ }^{*}$ University of New Hampshire, Durham, NH 03824, USA \\ ${ }^{\dagger}$ Jefferson Laboratory, Newport News, VA 23606, USA
}

\begin{abstract}
.
The CLAS is a multipurpose, large acceptance magnetic spectrometer, instrumented with detector systems sensitive to charged and neutral particles. The experimental program at CLAS is aimed at furthering our understanding of hadronic and nuclear physics, through electron and photon scattering experiments, which cover a large range of topics, including meson and baryon spectroscopy, nucleon structure through elastic and deep inelastic scattering, nuclear transparency, nuclear correlations and nuclear structure. This talk will briefly describe the detector and the collaboration that uses it and will highlight some recent results.
\end{abstract}

Keywords: CLAS Collaboration, electron scattering, DVCS, Generalized Parton Distributions, Hadronization, Color Transparency

PACS: 11.80.Cr, 11.80.Et, 13.30.Eg, 14.20.Gk, 25.30-c , 24.85.+p

\section{INTRODUCTION}

The Continuous Electron Beam Accelerator Facility (CEBAF) at the Thomas Jefferson National Accelerator Facility (TJNAF) in Newport News, Virginia, USA, provides a continuous beam of highly polarized (up to $85 \%$ ) electrons with energies up to $6 \mathrm{GeV}$ and at a current up to $200 \mu \mathrm{A}$ to the three experimental halls at the site. The photontagging system in Hall-B also allows for experiments with a real photon beam. The CEBAF Large Acceptance Spectrometer [1] (CLAS) is located in Hall B and has been operating since 1998. The spectrometer uses six superconducting coils to create a toroidal magnetic field around the target. Each of the six sectors of the spectrometer is instrumented with wire chambers, gas Cherenkov counters, scintillation counters and electromagnetic calorimeters, to detect and analyze both charged and neutral scattered particles. The spectrometer has an angular coverage for the polar angle, $\theta$, of $8^{\circ}$ to $140^{\circ}$, a momentum resolution of about $1 \%$ down to momenta of $150 \mathrm{MeV} / \mathrm{c}$ and can run at luminosities up to a few times $10^{34} \mathrm{~cm}^{-2} \mathrm{~s}^{-1}$ per nucleon.

The CLAS collaboration is a group of about 200 physicists from all over the world who have been using the CLAS for their experimental work. The experimental program with CLAS covers a wide range of topics in nuclear and particle physics, from the study of electromagnetic form factors of the nucleon, transition form factors, the baryon excitation spectrum and the search for missing resonances and exotic particles, spin structure of the nucleon, and the study of generalized parton distributions, to nucleonnucleon correlations in nuclei, the process of parton propagation and hadronization of struck quarks and the role of the nuclear medium in the modification of nucleon properties. The collaboration has published 96 physics papers and 17 instrumentation 
papers to date, which are available from the collaboration website ${ }^{1}$. This talk will highlight some of the recent results in Deep Virtual Compton Scattering (DVCS) in the next section and briefly describe an experiment measuring the properties of quark hadronization and the onset of color transparency in the next section.

\section{DVCS EXPERIMENTS}

The structure of the nucleon and how it is built up from its fundamental constituents, the quarks and gluons, remains an important area of investigation in Quantum Chromo Dynamics (QCD), the theory of the strong interaction. A fairly recent development in the description of the nucleon is the use of generalized parton distributions (GPDs), which provide a unifying framework to describe the nucleon structure. In addition to containing the usual form factors and parton distributions, the GPDs also reveal the correlation of the spatial and momentum distributions. For recent reviews on this topic see [2, 3]. One way to access the GPDs experimentally is through Deep Virtual Compton Scattering (DVCS) and variations such as Deep Virtual Meson Production (DVMP), Time-like Compton Scattering and Double-DVCS .

The CLAS collaboration has an extensive program measuring GPDs through the DVCS process. The first results were obtained from experimental data that was collected for other experimental efforts, however the versatility of the CLAS spectrometer allowed for the extraction of the DVCS beam spin asymmetry, which is sensitive to the interference term between the DVCS and Bethe-Heitler processes [4, 5]. Another experiment extracted the target spin asymmetry [6].

The addition of an extra calorimeter to the basic equipment of CLAS allows for a larger acceptance for the forward going photon in the DVCS process. This calorimeter is used by a number of dedicated experiments to measure the DVCS process. The E1-DVCS experiment [7] measured the beam spin asymmetry for a large number of kinematic points with very high accuracy [8]. More dedicated experiments have recently taken data, including the first experiment with a nuclear target $\left({ }^{4} \mathrm{He}\right)$ [9]. In the near future the Jefferson Laboratory accelerator will be upgraded to run at a maximum energy of $12 \mathrm{GeV}$ ( $11 \mathrm{GeV}$ for Hall-B), and an extensive program for additional experiments to measure the DVCS process has been proposed [10] using an upgraded spectrometer, the CLAS12 2 .

\section{THE EG2 EXPERIMENT}

The eg2 run period in early 2004 is a good example of how a single run period with CLAS can provide data for multiple experiments. In this case, the data was initially used for an experiment to measure the process of hadronization of a struck quark and a separate experiment to search for the onset of color transparency. Now, well after

\footnotetext{
${ }^{1}$ CLAS Collaboration Website: http://www. jlab.org/Hall-B/

2 The CLAS12 project, http://www.jlab.org/Hall-B/clasl2/
} 
the experiment was completed, additional analyses are starting up to look for nucleonnucleon correlations in the same data set and other analyses may follow.

A special feature of this experiment was that it used an electron beam of $5 \mathrm{GeV}$ and $4 \mathrm{GeV}$ on two targets simultaneously, which reduces the systematic errors when taking ratios of observables on different nuclear species. One of the targets was liquid deuterium and the other target was composed of a foil of either carbon, iron or lead. The targets can be clearly distinguished in the data analysis by use of a cut on the reaction vertex.

\section{Hadronization and Parton Propagation}

Hadronization is the process in which a colored parton (a single quark or gluon) transitions to a colorless hadron. Understanding parton propagation and hadronization is interesting in it's own right, and any improvement in our understanding of these processes would translate in an improvement in the tools used to determine the properties of the Quark Gluon Plasma produced in high energy ion collisions, and even help reduce the systematic uncertainties in neutrino experiments using nuclear targets. An excellent recent overview on the subject of hadronization and parton propagation can be found in reference [11].

In a simplified picture of the process of hadronization in semi-inclusive deep inelastic scattering (DIS), a struck quark transitions to a colorless hadron through the following steps. The virtual photon from an incident lepton is absorbed on a single quark inside a nucleus. The quark then propagates over some distance through the nuclear medium, possibly interacting through gluon radiation or other processes, and finally evolves into a color neutral pre-hadron, which subsequently emerges as a hadron. This process is highly non-perturbative and so cannot be easily described in QCD, so modeling and phenomenology are used to describe these processes.

Experimentally information about hadronization and parton propagation can be obtained by using the nucleus as a filter. By comparing particle production on several larger nuclei to that on a small nucleus like deuterium, information can be extracted about the relevant time scales for each of the steps in the production process. One such observable is transverse momentum broadening, $\Delta p_{T}^{2}=p_{T}^{2}(\mathrm{~A})-p_{T}^{2}\left({ }^{2} \mathrm{H}\right)$, the difference of the squared transverse momentum of a produced hadron on a large nucleus to that on deuterium. It is expected to be a good measure of partonic multiple scattering and is potentially associated with medium-stimulated gluon bremsstrahlung. An other observable is the hadronic multiplicity ratio $R_{M}^{h}$, which expresses the normalized change in production yield of hadrons in larger nuclei compared to deuterium or hydrogen. This ratio is sensitive to the formation lengths of hadrons. Experimental results have been reported by the HERMES collaboration [12] and CLAS collaboration [13, 14, 15].

A new experiment at an electron beam energy of $11 \mathrm{GeV}$ and using the new CLAS12 spectrometer is already planned [16] and will significantly expand on the the current measurements. 


\section{Color Transparency}

The same eg2 data set has also been analyzed to study the onset of Color Transparency (CT) in $\rho_{0}$ electro-production. CT is a prediction from QCD, first proposed in the early 80 s $[17,18]$, that point-like colorless systems will exhibit vanishingly small crosssections and are thus expected to travel through the nucleus with little attenuation. Such a Point Like Configuration (PCL) can be created in a scattering experiment at high momentum transfer $\left(Q^{2}\right)$. The onset of CT can be observed as a rise in the nuclear transparency, $T_{A}=\frac{\sigma_{A}}{A \sigma_{N}}$ with increasing $Q^{2}$. Many experiments have been performed to confirm this prediction, so far with mixed success, see one of the many review articles on CT for details $[19,20,21]$

The reaction used in the analysis of the CLAS data is incoherent electro-production of $\rho^{0}$ mesons off nuclei, which offers many advantages over experiments using a proton in the final state. It is expected that the onset of CT occurs at a lower $Q^{2}$ for a meson $(q \bar{q})$ compared to a hadron (qqq)[22]. Also, since the $\rho^{0}$ is a vector meson, the production mechanism is well understood by the Vector Meson Dominance model (VMD) as the fluctuation of a photon (which has the same quantum numbers as a vector meson) into a $(q \bar{q})$ pair. The photon at high virtuality $Q^{2}$ is expected to produce a quark pair with small $\sim 1 / Q^{2}$ transverse separation. CT then manifests itself as a vanishing absorption of this $(q \bar{q})$ pair as it propagates through the nucleus [23, 24].

The dynamical evolution of the small size $(q \bar{q})$ pair to a normal sized vector meson is controlled by the time scale called the formation time. In the rest frame of the nucleus this is given by $t_{f}=\frac{2 v}{m_{V}^{2}-m_{V}^{2}}$, where $V^{\prime}$ represents the first excited state of the meson and $V$ represents the ground state. This formation time needs to be sufficiently long to be able to observe CT.

A competing effect to $\mathrm{CT}$ in vector meson production is the coherence length effect, which is due to quantum coherence, the destructive interference of the amplitudes for which the interaction takes place on different bound nucleons. The coherence length effect depends on the coherence length, $l_{c}=\frac{2 v}{Q^{2}+m_{V}^{2}}$. This effect was recently measured by the HERMES collaboration [25]. Since this effect competes with the CT signal, it is important to hold the coherence length, $l_{c}$, constant. This was done in a later analysis of the same data [26], which shows a rise of $T_{A}$ with increasing $Q^{2}$, but suffers from poor statistics.

The analysis of the CLAS data used the reaction $e+N \rightarrow e^{\prime}+N+\rho^{0} \rightarrow e^{\prime}+N+$ $\pi^{+}+\pi^{-}$by detecting the scattered electron and the two pions which are decay products of the $\rho^{0}$. A cut was made on $W>2 \mathrm{GeV}$ to select the data above the resonance region. A cut on $t=\left(q^{\mu}-P_{V}^{\mu}\right)^{2}$ of $-t<0.45 \mathrm{GeV}^{2}$ selected the incoherent electro-production of the $\rho^{0}$. A further cut on the missing energy $\Delta E=v-E_{\rho}+t / M_{p}$ at $|\Delta E|<0.1 \mathrm{GeV}$ ensured proper exclusivity of the reaction. The resulting $\pi^{+} \pi^{-}$invariant mass spectrum shows a clear signal for $\rho^{0}$ production. The background processes to our main reaction are delta production and incoherent pion production. These background processes were modeled using the Genoa Monte Carlo generator from which we found the shapes in our spectrum. These processes were then included in the fitting procedure allowing only the relative strength to be varied. The $\rho^{0}$ signal was fit with a Breit-Wigner shape. The nuclear transparency was then computed by taking the ratio of the extracted number of 
counts for the nuclear target divided by the number of counts for the deuterium target and correcting for the difference in target thickness and number of nucleons, $T_{A}=C \frac{2 N_{A}}{A N_{D}}$.

A number corrections need to be made on the $T_{A}$ versus $Q^{2}$ graph. The correction for radiative effects is very small since it mostly cancels out in the ratio when computing $T_{A}$. A larger correction is due to pion absorption. When comparing with theoretical calculations, the decay of the $\rho^{0}$ into two pions and the subsequent absorption of of these pions in the nucleus or the target material must be taken into account. The $Q^{2}$ dependence of this process is very small, so the main effect of the pion absorption correction is to shift all the data points up by a constant amount. A more complicated correction needed to be made because of the combination of a finite acceptance of the detector, the kinematic cuts imposed on the data, the offset between the two targets and difference in Fermi motion between the two nuclear species. This acceptance correction was obtained by a very careful computation using an accurate event generator, which included Fermi motion for the nucleons, and an accurate computer simulation of the detector. Though this correction has a $Q^{2}$ dependence, we believe it is now well understood.

The final resulting $T_{A}$ for the data with the iron target were divided into 7 bins in $Q^{2}$ ranging from a $Q^{2}$ of $0.8 \mathrm{GeV}^{2}$ for the central value of the lowest point to $2.5 \mathrm{GeV}^{2}$ for the central value of the highest point. The $T_{A}$ exhibits a clear rise with increasing $Q^{2}$, far more than the statistical errors on the points. The resulting $T_{A}$ for the data on the carbon target also show a clear rise, albeit with lower statistical precision. If these results withstand the final tests of our analysis, this will be a very clear indication of the onset of Color Transparency.

A new experiment with CLAS12 has been approved, which will extend this study to higher $Q^{2}$, up to $7.5 \mathrm{GeV}^{2}$, and also cover a larger range of $l_{c}$ [28]. This experiment will obtain much higher statistical precision due to the higher luminosity capabilities of the upgraded detector. This will allow us to make a much more detailed study of the Color Transparency phenomenon and the process of vector meson formation and its interaction with the nuclear medium. This will enable us to study in far more detail how the point like configuration dresses with time to form the fully complex asymptotic wave function of the hadron, which puts us at the heart of the dynamics of confinement.

\section{CONCLUSIONS AND OUTLOOK}

The CLAS collaboration has an exciting physics program, of which this talk was only able to provide a very small glimpse, many topics were not covered. The versatility of the CLAS spectrometer allows for many different experiments with multiple physics observables extracted from the same data set. Now that a lot of data have been recorded, an active group of "data miners" is starting to analyze these experiments for observables that were not included in the original proposal. There are many opportunities, both in new experiments and old ones, for new students and researchers to join this effort.

In the near future, the CEBAF accelerator will be upgraded to $12 \mathrm{GeV}$ and the CLAS detector will be upgraded to the new CLAS12. With this a whole new era of experimental work at higher beam energies will start. 


\section{REFERENCES}

1. B. A. Mecking, et al., Nucl. Instrum. Meth. A503, 513-553 (2003).

2. A. V. Belitsky, and A. V. Radyushkin, Phys. Rept. 418, 1-387 (2005).

3. M. Diehl, Phys. Rept. 388, 41-277 (2003).

4. S. Stepanyan, et al., Phys. Rev. Lett. 87, 182002 (2001).

5. G. Gavalian, et al., Phys. Rev. C80, 035206 (2009).

6. S. Chen, et al., Phys. Rev. Lett. 97, 072002 (2006).

7. V. Burkert, L. Elouadrhiri, M. Garcon, R. Niyazov, and S. Stepanyan (co spokespersons), Jlab experiment e-06-003,

http://www.jlab.org/exp_prog/proposals/06/PR06-003.pdf.

8. F. X. Girod, et al., Phys. Rev. Lett. 100, 162002 (2008).

9. H. Egiyan, F. X. Girod, K. Hafidi, S. Liuti, and E. Voutier (co spokespersons), Jlab experiment e-08024, http://www.jlab.org/exp_prog/proposals/08/PR08-024.pdf.

10. A. Biselli, H. Egiyan, L. Elouadrhiri, M. Holtrop, D. Ireland, K. Joo, W. Kim, and F. Sabatie (co spokespersons), Jlab experiment e-12-06-119, http://www.jlab.org/exp_prog/proposals/06/PR12-06-119.pdf.

11. A. Accardi, F. Arleo, W. K. Brooks, D. D'Enterria, and V. Muccifora, Riv. Nuovo Cim. 032, 439-553 (2010).

12. A. Airapetian, et al., Phys. Lett. B684, 114-118 (2010).

13. W. K. Brooks, and H. Hakobyan, Nucl. Phys. A830, 361c-368c (2009).

14. W. K. Brooks, and H. Hakobyan, AIP Conf. Proc. 1056, 215-222 (2008).

15. W. K. Brooks (spokesperson), Jlab experiment e-02-104, http://www.jlab.org/exp_prog/proposals/02/PR02-104.pdf.

16. W. K. Brooks, G. Gilfoyle, K. Hafidi, H. Hakobyan, K. Hicks, M. Holtrop, K. Joo, G. Niculescu, I. Niculescu, L. B. Weinstein, and M. Wood (co spokespersons), Jlab experiment e-12-06-117, http://www.jlab.org/exp_prog/proposals/06/PR12-06-117.pdf.

17. S. J. Brodsky, "Testing Quantum Chromodynamics," in XIII International Symposium on Multiparticle Dynamics, 1982, edited by W. M. E. W. Kittel, and A. Stergion, World Scientific, Singapore, 1982, p. 963.

18. A. H. Mueller, "X," in Proceedings of the XVII rencontre de Moroind, Les Arcs, France, edited by J. T. T. Van, Editions Frontieres, Gif-sur-Yvette, 1982, p. 13.

19. L. L. Frankfurt, G. A. Miller, and M. Strikman, Ann. Rev. Nucl. Part. Sci. 44, 501-560 (1994).

20. P. Jain, B. Pire, and J. P. Ralston, Phys. Rept. 271, 67-179 (1996).

21. S. J. Brodsky, L. Frankfurt, J. F. Gunion, A. H. Mueller, and M. Strikman, Phys. Rev. D50, 3134-3144 (1994).

22. B. Blaettel, G. Baym, L. L. Frankfurt, and M. Strikman, Phys. Rev. Lett. 70, 896-899 (1993).

23. B. Z. Kopeliovich, J. Nemchik, A. Schafer, and A. V. Tarasov, Phys. Rev. C65, 035201 (2002).

24. B. Z. Kopeliovich, J. Nemchik, and I. Schmidt, Color transparency at low energies: Predictions for jlab (2007), hep-ph/0702272.

25. K. Ackerstaff, et al., Phys. Rev. Lett. 82, 3025-3029 (1999).

26. A. Airapetian, et al., Phys. Rev. Lett. 90, 052501 (2003).

27. B. Mustapha, and H. Lee, Private communication.

28. K. Hafidi, M. Holtrop, and B. Mustapha (co spokespersons), Jlab experiment e-12-06-106, http://www.jlab.org/exp_prog/proposals/06/PR12-06-106.pdf. 